\title{
Redesigning Staff Development as Organizational Case Learning: Tapping into School Stakeholders' Collaborative Potential for Critical Reflection and Transformative Leadership Action
}

\author{
Joseph Claudet \\ Department of Educational Psychology and Leadership, Texas Tech University, Lubbock, TX, USA \\ Email: joe.claudet@ttu.edu
}

Received October $2^{\text {nd }}$, 2012; revised November $12^{\text {th }}, 2012$; accepted November $21^{\text {st }}, 2012$

\begin{abstract}
This article reports on activities associated with one school-university partnership project focused on providing multimedia-integrated organizational learning and development opportunities to stakeholders in K-12 school communities struggling with real-world school change and improvement challenges. A central feature of the project's organizational case learning design is the use of multimedia case production technology in conjunction with context-specific school situational data to immerse school stakeholders in the theatrical portrayal and analysis of their own school dilemma challenges as a means to jumpstart collaborative teaming and data-driven problem solving. Multimedia case development and analysis efforts involving stakeholders in one middle school community who are confronting a difficult, politically charged set of school improvement challenges are highlighted. Project findings associated with the use of multimedia technology and data-driven organizational case learning as tools to inform an alternative staff development approach for promoting positive school leadership teaming and real-world problem solving among education stakeholders in K-12 school communities are discussed.
\end{abstract}

Keywords: Multimedia Case Development; Organizational Learning; Instructional Teaming; School Leadership and Improvement

\section{Introduction}

In my staff development and school improvement consulting work over the past two decades with K-12 schools and school districts, I have worked and continue to work to help educators and diverse groups of education stakeholders better understand who they are and where they are going as teaching, leading, and learning organizations through learning how to find creative ways to leverage their own situational contexts and challenges to work together more effectively as collaborative leading and learning communities. Leadership in school organizations is a very people-intensive enterprise. And leadership in schools (and, for that matter, leadership in any public service-centered organization) can often seem fraught with uncertainty due to the multiple, many times conflicting, views stakeholders tend to hold (fueled by their own passionate attitudes and beliefs) regarding pressing organizational issues and challenges. To help education stakeholders gain clarity amidst an array of diverse organization member perspectives and beliefs regarding who they are and where they are going collectively as organizations (i.e., to assist education community leaders in learning how to engage together in new ways to identify shared values and beliefs that can anchor and energize their collective school leading and learning efforts) I frequently adapt and apply insights from cultural anthropology and utilize phenomenological methods to help stakeholders gain new insights that can help them better understand their roles and responsibilities as members of communal leadership cultures.

There is a pivotal scene in James Cameron's groundbreaking futuristic movie Avatar (Cameron, 2009) that captures well the kind of intense organizational logjam that can occur when mul- tiple stakeholders harboring conflicting passionate perspectives and beliefs (starkly reflected in a conscious choice between pursuing "self-interest driven opportunism" or espousing "communal stewardship") collide head-on over organizational purposes and direction. The movie's storyline, set in the lush, faraway lunar environment of Pandora, portrays the intergalactic colonial expansion efforts of humans and their profit-hungry corporate sponsors in the early 22nd century who, as a result of having mined their own planetary resources to extinction, are now embarked on extraterrestrial exploitation of Pandora and its tribal, nature-respecting humanoid inhabitants, the Na'vi, to acquire new resources to fuel their unquenchable thirst for corporate profit. As the movie's plot line thickens and the perspectivist conflicts begin to boil over, one of the movie's primary characters, "Dr. Grace Augustine” (played by Sigourney Weaver), admonishes the corporate administrators in charge of the Pandora mining operation that "if you want to share this world with them [the Na'vi], you need to understand them [emphasis added]". With this statement, Grace Augustine makes a defiant claim for the importance of working collaboratively to strive to comprehend differences and to understand the multiple (sometimes conflicting) perspectives, values, and beliefs of the diverse stakeholders in your organizational arena. Comprehending differences and building multi-stakeholder understanding can be the key to fostering a climate of organizational trust and cohesiveness among multiple stakeholders and stakeholder groups. For Augustine, an interplanetary cultural anthropologist, understanding multiple stakeholder differences and then acting on those understandings are fundamentally important tasks essential to building a solid foundation for positive collaborative 
teaming. Interestingly, it was Grace Augustine and her fellow "avatar" scientists—along with "Jake Sully" (played by Sam Worthington), the story's "marine-turned-collaborative teaming” toruk makto [rider of last shadow] hero-protagonist-who, through working directly and continuously in a culturally immersive way via their "avatar bodies" with the native Na'vi, were the ones who were able to most easily realize and internalize these insights. Jake, in particular, through his own immersive avatar experiences living among and learning the ways of the Na'vi (i.e., understanding them), was able to enact his own personal transformation from a "self-interest driven opportunist" into a fierce advocate of "communal stewardship". Stated in organizational effectiveness terms, the key insight is: the organizational payoffs of collaborative teaming as a cooperative, communal stewardship-based "win-win" solution are more positive, desirable, and organization-enhancing than the not-so-positive end results of a competitive, opportunist-driven "win-lose" outcome.

Intriguingly, these insights on the nature of collaborative teaming and organizational effectiveness - specifically, the idea that nurturing multi-stakeholder trust and cooperation (and positive "win-win" organization-enhancing outcomes) can be best accomplished through helping diverse stakeholders who are harboring passionate multi-perspectivist differences build communal understandings as a foundational basis for generating a coherent, consensual team vision of organizational purpose and direction-became a central focus of my consulting work in one West Texas middle school community. As I was soon to discover, education stakeholders in this particular middle school were embroiled in an intense instructional change and improvement dilemma situation. And, these instructional improvement challenges, along with the multi-stakeholder perspectivist conflicts that were boiling over in their school community as a result of these challenges, were now straining these school stakeholders' collective capacities as organizational leaders.

\section{The Challenge of Instructional Change in a Middle School Community}

My ongoing school improvement consulting work in the West Texas Permian Basin region beginning in the early 1990s and now extending over two decades has led to the establishment of professional connections with a large number of campus principals and assistant principals, as well as central office administrative staff, in several school districts. So, I was not surprised when I received a call from a middle school principal in one of the Permian Basin region school districts in early fall of 2007. This principal indicated that she had heard of my consulting work with other schools in the area, and was wondering if she could talk with me about her own school situation and the leadership challenges she was facing. Intrigued by the principal's call and wanting to learn more, I readily accepted this principal's invitation to visit her campus and speak with her in person about her professional experiences and her current school leadership challenges.

I arrived at this West Texas middle school campus one morning in early October eager to meet the principal whom I had spoken briefly with earlier by telephone about the school leadership challenges she was facing. As I made my way to the school's administrative offices, I reminded myself-as my numerous experiences working with various other school communities in the region facing similar organizational change chal- lenges had taught me- - that I would need to talk with not only this school's principal, but with as many different school stakeholders as possible. I had learned through my own experiences over the years as a school improvement consultant that one of the best strategies I could employ to learn in depth about a school's overall organizational challenges would be to make an effort to become as familiar as I could with the views and beliefs of multiple stakeholders throughout the school community. It would be important for me to hear in stakeholders' own words their individual stories about their school community's teaching and learning challenges, and their own unique perspectives regarding their struggles with organizational change.

The principal welcomed me into her office and we sat down to begin our initial conversation. The principal indicated that she was in the beginning of her second year as principal of one of three middle schools in her district, and that the superintendent had specifically brought her in "to improve science and math scores" and "to turn this school around and make it a model of instructional transformation for other schools in the district to emulate". She also confided that she had been struggling for over a year now with what she believed were some heavily entrenched cultural and political views held by a number of influential education stakeholders within her school community-views which she believed were serving as powerful road blocks inhibiting and undermining her school change efforts.

As our conversation progressed, the principal shared with me her own realization that the true "complexity" of the challenges facing this school community had not become completely evident to her until she became fully immersed in the day-to-day leadership of her school during the fall of her first year as principal. It was during these first few months in her new position that she began to experience first-hand some of the entrenched school community political resistance to the district's new curricular and instructional improvement initiatives. In particular, in a targeted effort to improve student learning test scores, the district had just the previous year mandated the implementation of a new instructional teaming initiative - an initiative designed specifically to help ensure that the state's new curriculum and student learning assessment guidelines would be utilized consistently and effectively by teachers both within and across grade levels at all district campuses. This new district-mandated instructional teaming initiative would directly affect the way teachers throughout the district would now have to engage together in monitoring and utilizing their own classroom- and school-level data to inform their grade-level instructional planning.

The principal explained that science and math scores at her school were below proficiency and had remained so for quite some time. According to the principal, student performance in science and math has always been a central challenge at her school. The continuing regional demographic trend in recent years of an increase in Hispanic families moving into the area, however, has brought on additional new demands on her teachers in terms of meeting the instructional needs of an increasingly diverse student population. Her teachers' efforts over the past year have not resulted in any measurable performance gains, and her school has continued to languish as an underperforming campus. In addition to describing these escalating school performance challenges, the principal also shared with me some information regarding the professional staff makeup at her school. Many of the current teachers at the school have 
been teaching for fifteen or more years, and several of these teachers have spent the majority of their teaching careers at this middle school. In the past three years, though, as a result of the normal cycle of teacher retirements within the district, a number of new, early-career teachers have been hired and assigned to this middle school. These new teachers, although possessing comparatively less overall career teaching experience and less familiarity with the school and the community than the veteran teachers, have brought with them considerable energy and enthusiasm, as well as a variety of new teaching techniques and strong instructional beliefs which they are interested in both applying in their classrooms and sharing with colleagues.

The principal confided to me her strong belief that an energetic, school-wide embrace of instructional teaming has real potential for turning her school around, and she has been actively pushing the district-mandated instructional teaming initiative that began at her school during the past academic year. However, as teachers at her campus began to get involved in grade-level instructional teaming last year, it soon became apparent to this principal that the majority of her teachers lacked real understanding of the concept of "grade-level teaming", and lacked practical strategies on how to approach and go about it. Teachers at her middle school campus, the principal said, have also experienced real difficulties in effectively linking instruction across content areas-notably, in math and science, both of which are instructional performance areas the district central office is monitoring very carefully. And on top of these challenges, the principal added, the district is continuing its push to have teachers at each campus work proactively to more thoroughly "integrate" technology into instruction.

With anxiety in her voice, the principal explained that, based on her own continuing self-reflections up to this point on her collective leadership experiences at her school, she was now harboring a growing concern that many of the very same teachers who were passionately resisting the new instructional teaming initiative (which required that grade-level teachers learn how to work together as a team to examine and use their own students' performance data to make adjustments in their teaching and develop teaching interventions to better instructionally support these students) were, in fact, doing so because it was forcing them to get out of their well-established "comfort zone". Teachers were so resistant to the new teaming initiative, the principal conjectured, because it was abruptly challenging them to jettison a way of teaching (and a set of engrained instructional beliefs) that they had become very comfortable with over the years-teaching methods with which they were able to teach well those students who did not require any intervention. The principal further indicated that she felt that the instructional beliefs these teachers were espousing were, in large part, selfcongratulatory and self-corroborating, but did not match the new realities of their more diverse classrooms. These teachers in their own minds felt that they had been doing a very good job with their teaching, and that it was "these new kids and their home environments" that were the problem. Moreover, many of these same teachers were being supported in their convictions by a number of parents in the community (some with ties to school board members) who were also very content with the kind of "status quo" instructional methods being utilized by these veteran teachers. Some of these parents, in fact, were continuing to be quite outspoken and insistent in condemning the district's new instructional teaming initiative as simply "more unnecessary work for teachers". In addition to this, the princi- pal further explained, these entrenched teacher beliefs were being reflected as well in the kinds of school-level staff development recommendations that were emerging from the school's Campus Improvement Team (a group comprised of grade-level chairpersons and teacher representatives from the campus's seventh, eighth, and ninth grades), with the CIT prioritizing and scheduling staff development in-service events that focused on topics such as teacher stress and wellness, peer mediation, and assertive discipline. These kinds of topics, the principal surmised, might indeed offer some incremental professional learning benefits to teachers, but they did not appear to address in any substantive way any of the real, deep-structural instructional challenges the campus, and her teachers, were facing.

By now, the overall contours of this principal's story were sounding very familiar to me. Her story resonated with stories of organizational change that had been recounted to me over many years by other principals in schools throughout the region. Although many of the details of this principal's story were unique to her own school situation, there were many similarities with the school change challenges faced by other principals I had worked with. Of particular note was the fact that this principal, like so many other principals in similar situations, was confronting head-on the reality that the challenges of schoolwide instructional change do not stop at a school's front door and are not enclosed conveniently by a school's brick-andmortar walls. The process of transformative school change is one that involves an entire school community-a broad-based community to be sure that includes an array of diverse leading and learning stakeholders, including students, teachers, and administrators, as well as parents, school board members, and community business leaders.

As we wrapped up our initial campus conversation, the principal agreed with me that to gain sufficient information about her school's overall situation as well as specific, detailed information relating to the different grade levels on her campus (information which would be essential if I was to be genuinely helpful in my efforts as a school change consultant at her school), it would make sense for me to spend some time getting to know the school's teachers and other instructional staff in their own daily work environments. We both agreed that a great way to do that would be to spend some time sitting in on teachers' grade-level instructional planning meetings. Thus, with this principal's story of school change now firmly resonating in my mind, I spent the next three weeks in October sitting in on some of the weekly grade-level instructional team meetings that were now taking place on a regular basis at this middle school.

I knew from previous consulting experiences that spending time observing and listening intently to teachers as they worked during their daily grade-level team planning meetings was always an excellent way to: 1) get a sense of teachers' own attitudes and perspectives regarding the instructional challenges they and their school are facing; 2) glean first-hand from teachers their views on current school and district initiatives; and 3) begin to construct a composite picture of teachers' core beliefs about their overall professional work environments, their classroom teaching, and their students. As I quickly discovered, teachers at this middle school were very vocal and quite willing to share their views on their teaching experiences at this campus and the initiatives that were being implemented throughout the district which were directly affecting their professional work. In one eighth-grade team meeting I attended, teachers' genuine passion for teaching and their commitment to their students 
were clearly evident. As one eighth-grade teacher reflecting on his teaching experiences at this school for the past couple of years put it, "You know, I'm now beginning my third year of teaching, and some of us who've recently come to this school really understand the importance of collaborating to help kids see links between math and science, as well as make connections across other content areas, and we've tried working together to create lessons that combine concepts and challenge these kids, but many of these kids come to us with barely a fifth grade education, and on top of that, they're struggling with some basic ESL [English as a Second Language] reading comprehension issues". "Not only that", another team member interjected, "but in their computer classes they've learned how to download content off the internet, and they're turning that in as their work". She added, "I've been teaching at this school now for several years, and I've seen the diversity of our community increase. And, as a teacher, I continue to expend a great deal of effort to reach out to my students to help them be successful. But many of them simply don't have the language comprehension skills to be able to engage in the kind of critical thinking in science and math that the curriculum and the state assessments demand.”

In another seventh-grade team meeting I attended, teachers were openly questioning the appropriateness of the new instructional teaming initiative the district was implementing. As one teacher lamented, “. . . this new teaming initiative the district has mandated and our new principal is pushing - this is just more work for us to do. Some of us have been teaching for a long time and we pride ourselves on the quality of our teaching and the lesson plans we've developed over the years that guide our teaching". "Yes", echoed another seventh-grade teacher in the room, "I can not teach with someone else's plans". Another teacher on the team was quick to add her own perspective, “. . . this instructional team planning initiative really just creates another layer of work for us . . . we have to prepare additional 'team lesson plans' on top of our usual classroom plans. It just doesn't make any sense”. At this moment, a third teacher on this team, who had been sitting quietly at the planning table up to this point listening intently as her other colleagues spoke, sat up straight in her chair, eyeing her colleagues directly, and exclaimed, "Well, my friend's husband is on the school board, and she tells me that this instructional teaming initiative will be a topic of discussion at the next school board meeting. It's causing more work for us teachers, and the district is not even listening to what we need. The district is simply not providing us with the resources we need to work with these kids".

Many teachers within other teams I sat in on voiced similar perspectives about their district's improvement initiatives and the instructional challenges they were facing on their own campus. My collective experiences over a three-week period sitting in on a large number of grade-level team meetings with teachers at this middle school corroborated several of the instructional leadership concerns the principal had shared with me during our initial conversations. My experiences thus far at this campus also served to confirm my own growing suspicion that these "road blocks" to organizational change which were so evident in teachers' teaming conversations-viz., teachers' frustrations over having to implement the district's team planning mandate; the continued pressure teachers were experiencing in confronting the uphill challenge of trying to find suitable ways to respond to their students' diverse learning needs; along with my own perceptions regarding many of these teachers' apparent aversion to instructional risk-taking and their fear of leaving their instructional "comfort zones"-were, in fact, surface-level symptoms of a much deeper organizational dilemma challenge that this school community was facing. And this deeper, more systemic, dilemma challenge involved the need for stakeholders in this school community to learn how to come together in new ways to reinvent themselves as a middle school learning community through reassessing and redefining their core teaching, leading, and learning competencies and, in so doing, nurture a new organizational team learning culture that would enable these middle school stakeholders to reenergize and redirect their school improvement efforts.

\section{Expanding the Conversation to Find Common Ground and a New Understanding of the Purpose of School-Wide Staff Development}

The passionately held, varied perspectives on critical issues relating to instructional leadership I was hearing from teachers and the principal at this campus were indicative to me of a serious fragmenting of the organizational learning culture at this middle school-a rift in the overall fabric of stakeholders' collaborative organizational learning potential at this school that current instructional challenges coupled with district-instigated initiatives were causing to widen and deepen. At this point, the insights I was deriving from my collective consultant field notes along with the overall picture of instability that was forming in my mind regarding the instructional leadership and organizational learning dilemma challenges fomenting on this campus led me to schedule a further conversation with the school's principal. At this follow-up meeting, I pointedly informed the principal that, in my view, a number of critical factors were combining to create a perfect storm of organizational stagnation at this middle school. Most prominent among these critical factors were: 1) the school's teaching and learning history reflected in the campus's student performance data over the past five years; 2) the added pressures and political turmoil brought on by the district's current mandated change initiatives; and 3 ) the information I was collecting regarding the overall fragmented condition of the instructional leading and learning environment within and across grade levels at the school (evidenced by teachers' varied, deeply held, and often conflicting instructional values and beliefs concerning the possibilities for instructional change and renewal at their campus). Many teachers at this middle school were attempting to respond to their school's current challenges through a decidedly narrow-visioned and uni-perspectivist lens, making it difficult, if not impossible, for effective organizational change leadership to occur. The campus, in my estimation, was quickly heading toward a critical impasse-one that could only be addressed by dramatically opening up and deepening the organizational conversation.

At this point, I briefly shared with the principal my experiences in using organizational futuring techniques to assist school community stakeholders at other regional campuses who either had been or were now grappling with similar dilemma challenges. One particular applied form of organizational futuring that is employed regularly in business organizations and which I've utilized in a number of school settings over the years involves the use of future search conferences (or, future searches) as a means to engage large numbers of school stakeholders in coming together openly as an organizational com- 
munity to: 1) critically examine their own school's organizational history; 2) listen intently to multiple stakeholders' individual beliefs and perspectives; and 3) explore their school's current challenges from multiple angles. Participants in these future search conferences typically include 30 to 65 or so stakeholders from across the entire school community organization-including campus-level teachers and administrators, parents, district central office personnel, and community business leaders. One important goal of the future search conference design is to “. . . explore and validate differences, but we don't 'work' them. Should people open old wounds, fight old battles, or jump to problem-solving, we seek to have them acknowledge each other's reality and remind them that the task is finding common ground and future aspirations [emphasis added] . . . When we work on common ground and common futures, we tap deep wells of creativity and commitment” (Weisbord, 1992: p. 6).

If carefully planned and implemented, future search conferences can be a powerful means to assist school stakeholders in gaining needed clarity as a broader organizational community on complex dilemma challenges they are presently confronting-including helping to raise organization members' own awareness of the multiple, conflicting stakeholder perspectives that are, very often, directly contributing to the multi-perspectivist logjam of values and beliefs that can be fueling their school's dilemma situation. Importantly, stakeholders' involvement in the future search conference process can potentially help to highlight and crystallize organization members' critical understandings of fundamental values and beliefs that they can agree they share with each other. These shared understandings can then provide a "common ground" or communal perspective upon which stakeholders can then begin to work together to fashion a new collaborative vision of a desired positive future for their school organization.

With the principal's enthusiastic assistance, three future search conference meetings were planned and conducted at this middle school in the early spring of 2008. Participants in the future search meetings included current members of the school's Campus Improvement Team (i.e., the three grade-level chairpersons, one for each grade level—seventh, eighth, and ninth grades; two teacher representatives from each grade level; and the principal and assistant principal). On my recommendation, this core group of stakeholders was expanded substantially to include eight teacher representatives from each of the seventh, eight, and ninth grade-level teams, as well as several additional parent participants from each grade level, along with a number of district central office personnel. The structure of each future search meeting included multiple small-group breakout sessions followed by full-group debriefings and communal discussion. This structure was utilized to encourage participants to intently listen to and to strive to better understand their fellow stakeholders' perspectives on issues of importance, and to share commonalities as well as differences in individual thinking.

Participant interactions during each of the future search meetings served to underscore the breadth and depth of differences existing across stakeholders and stakeholder groups on challenging issues affecting this middle school campus. For example, at one of the full-group debriefing sessions during the second future search meeting, several different stakeholders expressed views that starkly highlighted their own uniquely individual perspectives on their school's instructional chal- lenges, while also serving to frame in broad strokes the nature of the underlying conflict existing among stakeholders' fundamental teaching beliefs. In particular, several of the same instructional attitudes and beliefs I had heard from teachers during their daily teaming meetings (i.e., teachers having to deal with the added pressures of trying to respond to the needs of English language learners, coupled with real technology issues and multiple content area integration challenges) were expressed again by more teacher participants during the future search sessions. As one teacher summarized, "The increasing diversity of our kids continues to make teaching much harder. Many of our students are performing well below grade-level in reading comprehension, which makes teaching content and reaching the state's student performance objectives much more challenging. I feel like we're already being pushed to the limit with all the district's school improvement demands. And now comes this added burden of this new teaming initiative." The school's assistant principal, who was listening intently to this teacher's comments, responded, "Well, our test scores have to improve. And a way to do that is to start focusing on examining and leveraging our own grade-level data so we can make needed adjustments in our team instructional unit planning to better meet students' needs”. The school principal immediately followed up on the assistant principal's remarks, "This 'teaming initiative' is not about working harder. It's about doing things differently from how we've done them in the past. We all agree that our student population is changing, becoming much more diverse, and because of this we also need to acknowledge that we need to find new ways to change our teaching strategies to meet our changing students' needs. Rather than thinking of these new challenges as an impossible burden, we can leverage this as an opportunity to engage in new kinds of team-initiated and datadriven progress monitoring to help all of our students succeed”.

Several teachers sitting near the principal noticeably bristled at the principal's statements, and the tension in the meeting room was now palpable. There was an awkward silence as teachers across the room exchanged pointed glances with one another until one of their colleagues, a veteran teacher at this middle school, sat up straight in her chair and firmly declared, "Well, some of us have been teaching at this school a long time, and we feel that we've been working very hard over the years to meet our students' needs and that our teaching overall has been pretty successful”. The principal stood up and slowly surveyed her teachers seated throughout the room, then she spoke resolutely, 'I'm not questioning how hard you've been working, and I am absolutely sure of your dedication to your students and to our school. But, if we are to improve as a school community, and if we are to improve in the performance areas that we need to address, we will need to learn how to work differently. Our teachers in many ways have been working in isolation and, because of this, many of our students are not making needed connections across the curriculum, particularly in science and math".

During the third and final day of future search meetings, as school improvement consultant I took a few minutes at the outset of the final full-group session to congratulate stakeholders on their candidness and energetic participation throughout these meetings. I also reemphasized to all participants that one of the central purposes of the future search endeavor is to help organization members openly explore each other's values, beliefs, and perspectives in a nonjudgmental, panoramic way as an organizational community. And, further, that this process 
can be used as an important opportunity to discover common threads that can potentially weave through multiple stakeholders' sometimes disparate beliefs_-shared understandings that stakeholders might be able to construct as a group that could bind them together as a teaching, leading, and learning organization. These shared understandings, if effectively internalized, would then serve as a valuable "common ground" upon which stakeholders could begin the process of collaboratively envisioning their desired organizationnal future.

To facilitate the summary communal sharing process at this final full-group session, a participant scribe from each morning session breakout group reviewed their group's compiled list of "organizational issues, challenges, and constraints" which each focus group had generated. As each focus group scribe shared their list with the full group, a composite picture of this middle school organization, including stakeholders' collective sense of their school community's pressing organizational challenges, quickly emerged in full view. The aggregate list of challenges that emerged highlighted the school's (and district's) expanding population diversity, the test score deficits in science and math, concerns over how to best go about integrating technology into the curriculum, the growing pressures to meet the state's student performance standards, and the district's multiple program mandates, particularly the instructional teaming initiative. An important additional organizational challenge the middle school future search participants now agreed also belonged on their "challenges list" was: the clearer sense of urgency these school leaders now shared as a group in needing to find some creative way to break through their multiple perspectivist gridlock to move their school community forward.

Just as the scribe of the final focus group wrapped up her summary presentation to the assembled stakeholders, one of the middle school parents in the room turned to address the group. "I see that the challenges facing our school community are very real”, the parent began. "And I can also see that as a group we can agree that there are no easy solutions to these challenges. What I do know though is that I want my kids to learn relevant content in science and math, and in other areas. But I also want them to obtain useful technology skills so they can be productive in the real world. I know my kids take computer classes, but I'm not sure they're necessarily acquiring specific skills on how to utilize this technology in ways that actually enhance their learning of science, math, geography, etc. When I ask my kids what are you learning in computer class that helps you better understand math and science problems, they can't tell me. My kids have all become very attached to their ipods, their iphones, and their ipads. But are they using these devices simply to socialize and play online games, or are they using them as real tools for learning?” The other stakeholders in the conference room were listening intently as this parent continued, "You know, I think teachers at this school have a responsibility to work together within all learning areas to teach kids how to leverage technology to help them connect the dots across the curriculum, across grade levels, and across the street to the real world! I understand teachers' concerns about how their classrooms have become more diverse and that this makes it more challenging to teach. But diversity is part of today's real world, and it seems to me that technology can be a genuine learning tool that can open up that world to all of our kids.”

Following this parent's remarks, a central office curriculum supervisor then stood up, smiled and nodded at the parent in appreciation of her comments, and stated, "We are very aware that the diversity of the district's student population has been expanding and that the students in our classrooms today are different in important ways from the students we were serving ten years ago, and this reality does create new teaching and learning challenges for all of us in the district. And, at the central office, we are fully aware that many of our veteran teachers have been complaining that they feel stretched to the limit and are already doing everything they can within their individual classrooms to meet these new instructional challenges. But, you know, sometimes how we approach a challenge ends up defining that challenge for us. Many of our teachers see this increased diversity as a constraint, but maybe-just maybe-this new 'challenge' might also harbor some hidden opportunities that we can capitalize on, if we can just learn to view these challenges in new ways.” The curriculum supervisor's comments were followed by a few moments of silence among the middle school future search participants as they sat together at their focus group tables arrayed around the conference room, as stakeholders appeared to take some time to reflect on what had just been said.

By the end of this final session, stakeholders participating in this series of future search meetings were willing to admit that as a school and district learning community they had reached an impasse and had exhausted their ability to generate creative ideas on how to deal with what appeared to be a genuine dilemma situation at this middle school. For the future search participants in the room, the outlines of their middle school's dilemma were now clear: how do we respond to teachers' instructional teaming implementation concerns and also effecttively address our diverse students' teaching and learning needs, improve our science and math scores, and move our school community forward? As this middle school campus's school improvement consultant, I was intent on helping these stakeholders expand their thinking and find workable solutions to their organizational challenges. So, as I had done in the past at other campuses in the region, I used this "reality moment" as an opportunity to present a new idea to these stakeholders. I proposed to these stakeholders that they combine the numerous insights about technology, teaching and learning diversity, and collaboration they had collectively generated during their future search conversations to explore a new way of engaging with their dilemma situation that they had not yet tried. Rather than fretting over the constraints of technology, why not harness technology as an organizational learning tool that stakeholders themselves could use to collaboratively examine anew their own situational challenges and, in doing so, possibly generate new kinds of creative action strategies that they had not as yet envisioned?

In short, I proposed to these stakeholders that they embrace an alternative, school-wide staff development project for the next two years-to engage in organizational learning in a new way through working together as a "multimedia production team" to develop and produce a multimedia case story about their own middle school instructional leadership challenges.

\section{Embracing a Multimedia Case Development Opportunity and a Renewed Interest in Organizational Learning}

With some anxiety about the unknown, but with a determination to move forward, stakeholders at this middle school agreed to work with my university colleagues and myself in a multi- 
year project to explore their school improvement dilemma challenges as a multimedia case development team. Case development work undertaken at this campus constituted part of a larger organizational case learning research and development effort conducted in a number of schools and school districts in the West Texas Permian Basin and Texas Panhandle regions in conjunction with a decade-long research and development project made possible through initial funding in 1996 through 1998 (totaling US \$400,000) provided by the Sid W. Richardson Foundation (Fort Worth, Texas), the Abell-Hanger Foundation (Midland, Texas), and the Franklin Charitable Trusts (Post, Texas). This initial funding supported the creation of a multimedia case simulation research and development lab in the College of Education at Texas Tech University that served as headquarters for a number of university researchers and multimedia specialists who collaborated with regional school stakeholders on individual project cases. University-based R\&D multimedia lab specialists with technical expertise in using broadcast-quality betacam SP cameras, sound mixing equipment, and digital nonlinear video and audio editing system hardware and software played essential roles as members of a university-based production team who worked in tandem with regional, campus-based case development teams within the project's overall university-K-12 school collaborative partnership design.

Case development work at this middle school was conducted over a two-year period beginning in late spring of 2008. The school's case development team was comprised of a majority of the same stakeholders who participated (as members of the school's expanded Campus Improvement Team) in the threeday future search events held earlier in the spring. An important aspect of preliminary case development work carried out by stakeholders at this middle school involved case team members directly in conceiving and storyboarding individual case scenes that accurately portrayed key events and interactive dimensions of the case situation. Case team members were encouraged to craft individual scenes that highlighted encounters between multiple stakeholders holding conflicting beliefs and perspectives on critical case issues in order to bring into sharp focus specific elements of the school's overall dilemma situation. Following completion of this storyboarding process, team members then engaged in the detailed work of carefully scripting each individual scene. Because of the entrenched political tensions surrounding this case situation that were so pronounced within this middle school community (tensions that were to a large extent also reverberating throughout the wider school district community), case team members spent a good deal of time working on the scripting of case scenes to make certain individual scenes were accurate in capturing the nuanced perspectives of individual stakeholders and stakeholder groups. Refinement work on case scene scripts was completed in May. The combined multimedia case team consisting of middle school stakeholder case developers and university multimedia production specialists then began in earnest to plan filming activities for the summer.

One common thread linking case development work at this middle school with other work completed over several years at other campuses participating in funded regional case project activities was the critical reflective immersion aspect of stakeholders' involvement in case development and refinement activities. The entrenched school-community political tensions that were such a pronounced feature of this particular case made this immersive element especially important as university production team members assisted middle school stakeholders in refining case scene scripts. Special attention was given to ensuring that relevant stakeholder perspectives on key case issues were accurately portrayed in the scripted interactions occurring within individual scenes. This attention to perspectivist accuracy - and, in particular, to encouraging stakeholder team members to cultivate a sustained critical reflective stance toward each others' deeply held organizational perspectives and beliefs-spilled over and emerged anew in dynamic ways during the actual filming process. During case filming activities, school stakeholder team members were directed to assume and play different "organizational roles" than the ones they played in real life for the filming of individual case scenes. This active role swapping and intense immersive experience in playing other stakeholders' roles during case scenes filming work (an organizational team learning strategy that was included intentionally as an important aspect of overall case filming activities) forced stakeholders to spend time consciously examining their colleagues' own (often different) perspectives and beliefs regarding critical organizational issues surrounding the case.

Often during scene shoots, individual stakeholders would become agitated and call for a time-out. These stakeholdersturned-actors would then complain that their own real-life role perspectives and beliefs were not being portrayed accurately by their actor-colleagues during scene interactions (through appropriate vocal emphases, facial expressions, body language, etc.). During the time-out, stakeholders would then proceed to coach their fellow actors on the nuances of their own real-life role perspectives so that their perspectives and beliefs about case issues would be portrayed by their colleagues more accurately and persuasively during filming. This kind of spontaneous stakeholder multi-perspectivist peer coaching became a common occurrence during scene filming — so much so that the university production team made it an ongoing practice of filming and capturing these time-out peer coaching sessions as an additional and important organizational learning dimension of case development activities. Many of these impromptu stakeholder peer-coaching sessions were then incorporated (as organizational learning archive elements) into the final multimedia case's interactive design.

The collaborative project team of middle school stakeholders and university production specialists worked enthusiastically on completing filming of case scenes and other related interactive/reflective elements of the multimedia case during the summer and following fall. Regional education service center consultants having expertise in middle school instructional leadership and organizational change were also invited to become members of the case development team and participated during the following school year in collaborative analyses of the case and in filming reflective discussions with school stakeholders about various aspects of the school's organizational challenges depicted in case scenes and related case databases. These filmed reflective sessions about this middle school's instructional dilemma were also integrated into the overall multimedia case design.

\section{Multimedia Case Design Features}

A number of interactive navigational features were incorporated into the overall design of the multimedia case to enable case team developers and users of the multimedia case to di- 
rectly engage with and analyze multiple aspects of the organizational case situation. These design features provided a context-specific, multimedia organizational learning environment within which middle school stakeholders could: 1) collaboratively examine in detail multiple dimensions of their dilemma situation; 2) access and review multiple performance databases and information resources relating to the case situation; and 3) propose and reflect on the merits of various kinds of school improvement action strategies that might best address their school community's organizational leadership challenges. The interactive features of the multimedia case design were structured so school stakeholders could examine the case scenes and other case databases both individually and as a group.

Key features of the project's multimedia case design template are illustrated in Figures 1 through 4. The multimedia case design utilizes a "school leadership office" visual metaphor as the primary interactive interface within the overall organizational learning environment (Figure 1). This interactive environment consolidates and makes available a variety of interactive school information databases and professional resources for easy access by users (e.g., student demographics, multi-year school performance and accountability data, school district policy handbooks, state and national school leadership performance standards, etc.). Case users (working individually or as a team) can navigate this interactive multimedia environment to: 1) access and view individual case video scenes; 2) examine specific school leadership state and national professional performance standards relevant to the case; 3) interact online with educational colleagues and mentors (e.g., school district central office curriculum directors, program supervisors, and other personnel; regional education service center curriculum and instruction consultants; state education agency personnel; university professors); 4) search case-specific information databases and educational resources contained in digital file folders accessible within the multimedia environment; 5) develop, refine, and digitally archive their own critical reflective analyses of interactive case scenes (and/or targeted video frame segments of individual case scenes); 6) engage with school community colleagues to discuss the relative merits of various proposed action strategies to address case challenges; and 7) formulate data-driven sets of short- and long-term school improvement action plans.

A central area within the multimedia case's organizational

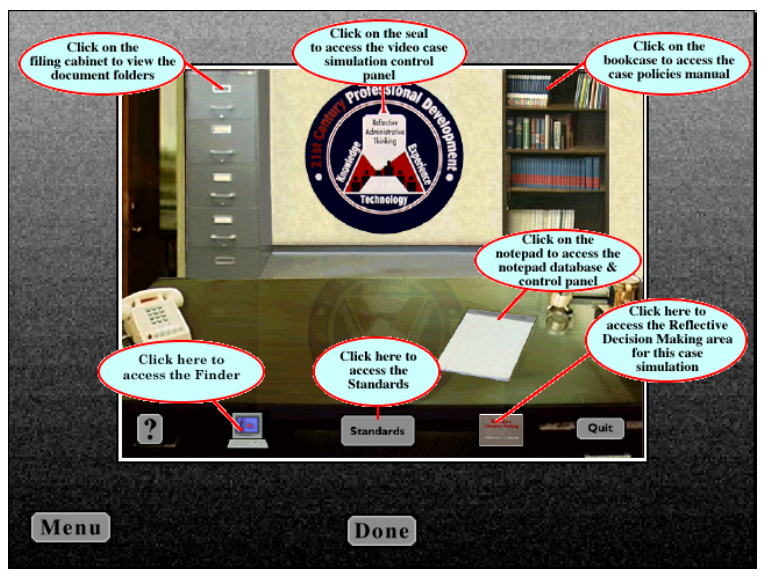

Figure 1.

Multimedia case "school leadership office" environment and multiple database interface. learning environment is the Case Video Scenes Database (Figure 2). Navigating within this database, users can access, load, and view individual digital video case scenes. Each scene portrays one or more interactive critical incidents involving various role players in the case dilemma situation. To facilitate critical analysis of interactive dimensions of the case situation, users can utilize the special "video mark" frame analysis capability included within the multimedia case design. The "video mark" function allows users to select and digitally mark specific sections of individual case video scenes for further analysis (Figure 2). Using this "video marking” tool, users can digitally frame, review in-depth, and develop written critical analyses of selected sections of various case video scenes. The "video marking” capabilities contained within this Case Video Scenes Database area are especially useful in providing users with the opportunity to zoom in on individual scene details and carefully examine specific role player interactive dynamics associated with various stakeholder perspectives contributing to the overall case dilemma situation.

Users can conduct their video scene frame analyses through focusing on individual "critical incidents" involving multiple stakeholder perspectivist conflicts identified within individual scenes, as well as also engage in comparative cross-scene analyses of multiple critical incidents occurring at different times in the overall case situation timeline and perhaps involving a variety of different stakeholders and/or stakeholder groups within the multimedia case design's Reflective Analysis area (Figure 3). To further enhance informed analysis and group discussion of case dilemma challenges, users can utilize the multimedia functions available in the Reflective Analysis area to sort and organize their collective "video marks" and accompanying written critical analyses into specific cognitive domain areas (i.e., programmatic, contextual, functional, interpersonal) referenced in available state and national school collaborative leadership performance standards. This analytic sorting process highlights specific collaborative leadership domain areas that are most clearly and consistently referenced in users' collective "video mark" written critical analyses, and enables users to engage in focused professional standards-informed group discussions of critical aspects of organizational change leadership that are of central relevance to the case situation. As users are involved in this ongoing process of developing, refining, and discussing their "video mark" frame analyses, they have access to all case-relevant information available in the digital file da-

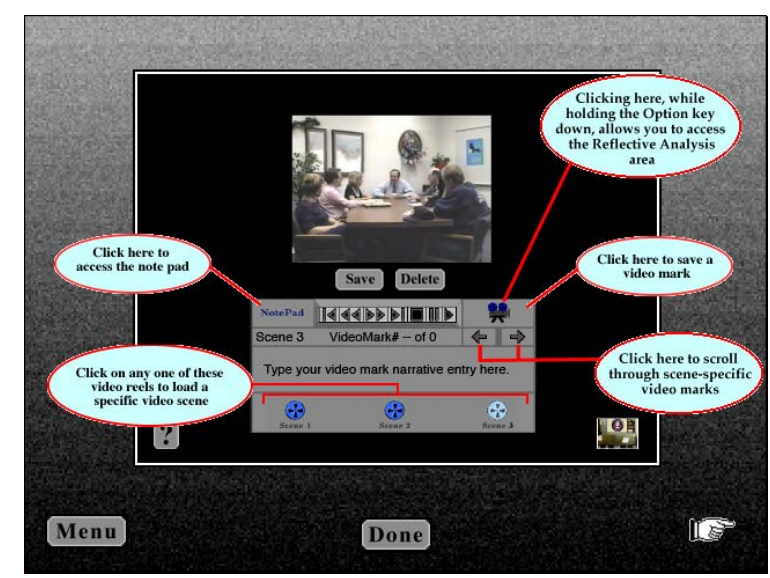

Figure 2.

Case video scenes database area with "video mark" capability. 


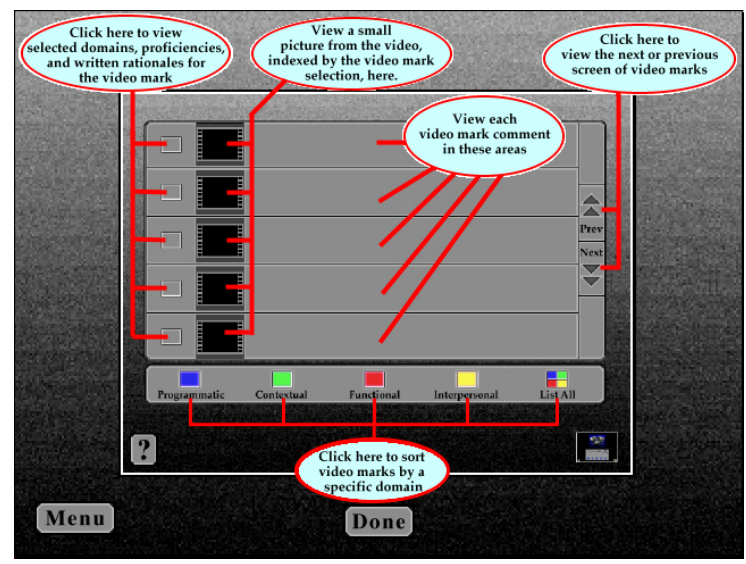

Figure 3.

Case reflective analysis area illustrating "video mark" comparative cross-scene analysis and leadership performance standards sorting functions.

tabases included within the multimedia case environment (e.g., school community demographics; grade- and school-level student performance and accountability data; school district policies and program resources; state and national school leadership standards; expert panel perspectives; etc.). In addition, the overall case analysis design allows users to digitally archive their individual- and team-developed "video mark" narrative analyses and group discussion notes in the multimedia case's organizational learning program for future reference.

Case simulation users can reflect on additional school leadership insights pertaining to the case situation through accessing the case's Reflective Decision Making area (Figure 4). This area presents a number of short "expert panel” video sequences featuring discussions by seasoned school community leaders and educational consultants on specific organizational issues, stakeholder multi-perspectivist dynamics, and instructional leadership challenges highlighted in the case simulation. Case team members, as well as school stakeholders in other schools and school districts interested in the leadership challenges profiled in this middle school case, can review these expert panel video sequences to obtain additional insights to inform their own real-world collaborative leadership thinking.

The above interactive design features were incorporated into the multimedia case to stimulate middle school stakeholder project participants to: 1) focus in directly on the multi-perspectivist issues and challenges contributing to their middle school dilemma situation; 2) leverage their own reflective thinking and group analyses to reframe their dilemma and develop viable sets of short- and long-term action strategies; and 3) through engaging in the overall multimedia case development and analysis process, generate new insights as a multimedia team on the potential benefits of embracing a collaborative teaming approach to organizational problem solving. Collectively, these multimedia case design elements provided case team members with an interactive digital platform to explore and analyze their own organizational dilemma situation in new ways and to engage in reflective conversations on how to effectively move their school community forward. This middle school instructional leadership case simulation was incorporated as one new installment into the growing body of multimedia cases that were developed and disseminated to school districts throughout the region as part of the project's multi-year school leadership case development activities.

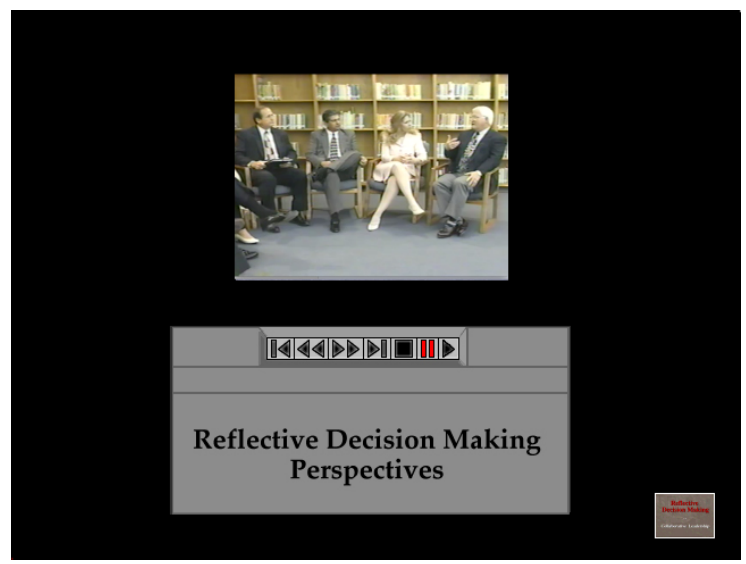

Figure 4.

Case reflective decision making area presenting a number of expert panel case perspectives.

\section{Discussion}

Collective experiences of middle school stakeholders and university-based multimedia production specialists working together over a two-year period in this collaborative project served to generate a number of intriguing insights concerning the challenges and possibilities of organizational learning and development in this middle school learning community. In particular, these insights related to the potential usefulness of multimedia case development as an alternative staff development endeavor that can help foster among diverse school stakeholders a strong team mind-set and a collegial desire to realize positive school community instructional change and organizational learning. Some of the insights gleaned from collaborative project work conducted at this middle school are highlighted and discussed below.

\section{Multimedia Case Development Activities Were Found to Be Useful as a Creative Means for Energizing School Stakeholders' Collective Potential for Renewed Organizational Learning}

The immersive "production team" learning environment aspect of multimedia case development activities was found to be a useful means for helping stakeholders develop a new, more informed organizational mind-set regarding the possibilities for instructional change and improvement in their middle school community. Importantly, participation in project activities enabled stakeholders collectively to evolve and crystallize new organizational perspectives on the potential benefits of thinking differently and working together in new ways as a multimedia case production team. Through their involvement in case development and refinement activities, school stakeholders learned from each other about how to creatively leverage the intensive collaborative scrutiny of their own context-specific dilemma situation itself as a creative means to examine anew their organizational challenges from more integrated vantage points. In doing so, case team members were able to glean new insights about instructional leadership and to discover new ways to enact positive organizational change and move forward together as a school community. The kind of immersive, collaborative inquiry process engaged in by middle school stakeholders 
participating in this multimedia case project reflects in positive ways the research-based recommendations of many current organization thinkers focused on the challenges of system-wide strategic change who emphasize the need for collaborative leaders to work together to integrate multiple perspectives in order to yield deeper understandings. As Jacobs (1997) states, "We need to integrate other viewpoints and perspectives with our own by really listening and seeking to understand realities different from those we currently believe in order to see the world in more whole, broad, and often less absolute ways. Subsequently, we act differently based on these insights." (Jacobs, 1997: p. 101) Additionally, the context-specific and dataintensive reflective analysis design of the middle school multimedia case project reported herein is consistent with recent emphases in the school leadership literature on the importance of making data-driven collaborative inquiry an integral part of school operations and improvement initiatives (Love, Stiles, Mundry, \& DiRanna, 2008: p. 30).

The intensive multimedia case development and analysis process engaged in by university and school-based collaborative team project participants at this middle school (as well as at other schools in the region over a period of several years) is grounded firmly in a phenomenological approach to organizational learning (Van Manen, 1990). A specific overarching theme running through all phases of the organizational case learning activities team members participated in during the two-year middle school staff development and case production efforts detailed in this report involved stakeholders critically examining and leveraging their own lived experiences as a powerful means for gaining new insights about both themselves and their organization's challenges (Smith, Flowers, \& Larkin, 2009). Collective experiences of collaborative teams of researchers, multimedia production specialists, and school leaders working over multiple years on case project efforts in schools like this one have resulted in the accumulation of a great deal of positive evidence to support the claim that school community stakeholders, in fact, learn best about how to effectively lead their schools in context and from each other. In addition, the evidence also suggests that involving stakeholders in an immersive, constructivist multimedia learning environment that challenges participants to step out of their comfort zones and carefully examine their own context-specific dilemma challenges in new, multi-perspectivist ways (a kind of virtual team learning experience) holds potential as a creative tool for invigorating stakeholders' interest in and commitment to their own realworld instructional teaming and related, ongoing organizational leading and learning efforts.

Campus improvement team members' unique involvement as a case production team in the multimedia case development and analysis process engaged in at this middle school helped bring into clear focus important aspects of organizational learning and served to highlight critical organizational core competencies that, up to this point, had remained predominantly dormant in these middle school community members' collective organizational leadership practices. The intensive experience of being immersed and fully engaged for an extended period of time in the interrelated facets of multimedia case development (e.g., filming various case scenes, reviewing and organizing instructional performance data for inclusion as databases within the multimedia case, and working together as a group to analyze critical interactive incidents between and/or among multiple stakeholders and stakeholder groups occurring within the over- all case situation) caused stakeholders to begin to reflect with new insight on the multiple, interrelated dimensions of their school community instructional dilemma. In particular, case team members' sustained collaborative efforts in analyzing various stakeholder role interactions and multi-perspectivist conflicts highlighted during multimedia case scene production activities caused team members to begin to actively scrutinize their overall dilemma situation with an eye to more critically elucidating the multi-leveled dimensions of their dilemma situation. This involved stakeholders, initially, in focusing their efforts in analyzing the surface-structure features of their dilemma challenges (including the array of organizational issues informing their dilemma situation and the immediate leadership challenges these issues presented). These initial team analytic efforts, in turn, became a catalyst for jumpstarting stakeholders' enthusiasm for wanting to engage in further focused group discussions to attempt to discern what might be the underlying root causes of their dilemma (specifically, to answer the fundamental, deep-structural analytic question: How did we get to this state of affairs as an organization?). And, importantly, engaging in this kind of organizational sense making process empowered stakeholder participants to examine anew their collective potential for organizational resilience and served to reenergize stakeholders' shared sense of "what is possible” in terms of their communal capacity to reinvent themselves as school leaders. These latent core competencies (in-depth group dilemma analysis and sense making; organizational resilience; and a renewed, shared capacity for collective reinvention) gradually emerged and became evident to university specialists observing and assisting middle school case team members during case project activities, and were increasingly recognized and acknowledged by stakeholder participants themselves as they continued to work together within case production activities.

\section{The Case Development Project's “Collaborative Teaming” Design Served as an Impetus for Stakeholders to Adopt a Distributed Leadership Stance toward Organizational Change}

Cultural anthropologists have documented that, in some cultures, communities of people confronted with immediate productivity challenges are able to successfully navigate those challenges through tapping their own culturally ingrained, natural predilection for collaborative problem solving and organizational learning. In Japanese culture, for example, the Japanese people possess a well-developed social disposition toward collaborative teaming and focused group problem solving. This inclination stems from the nature of life in rural Japanese communities that has centered for centuries on subsistence rice farming. For example, when confronted with a real and immediate organizational problem affecting their small community's rice productivity, such as an overgrowth of weeds in their rice fields' water canal irrigation system, the inhabitants of any rural Japanese farming community will instinctively come together as a group (with both male and female community members involved equally in the discussion) to articulate the problem's parameters and carefully deliberate the relative merits of possible practical solutions. The group will then proceed to implement a final agreed upon set of action strategies only after working through any differences and reaching complete unanimity within the group on the superiority of this final set of 
action strategies in comparison to all others considered. The Japanese refer to this group thinking and decision making process as kaizen (Seymour, 1995).

This process of kaizen (i.e., responding directly to encountered problems through careful group deliberation followed by practical decision making and resolute action) has become historically ingrained in the Japanese cultural psyche over many centuries and can be defined in organizational productivity terms as "the ongoing pursuit of continuous improvement by every organization member". This emphasis on the ongoing pursuit of continuous improvement by all stakeholders involved in an organization distinguishes kaizen as an "eastern" cultural practice from many other "western" cultural practices, such as organizational practices in America and other western countries, which have tended (at least historically) to value specialistdriven innovation by one or a few organization members over general group collaboration. In Japanese culture, in contrast to western cultures, willful individualism and competitiveness are not valued modes of behavior. Careful discussion leading to decision-making unanimity followed by group action is how most major jobs get done. For the Japanese, relationship to the group is of overwhelming importance in everything they do. Within Japanese rural farming villages, community members' own survival and continued productivity provided the strong impetus for developing this distinctive culture of collaborative teaming. Through their ongoing, collective experiences in confronting and solving many real-world problems through group deliberation and action, the Japanese over time have internalized this teaming process as a valuable and useful part of their cultural mores. Moreover, this centuries-old cultural inclination towards collaboration and group problem solving has transferred readily to both the shop floor and the boardroom to positively impact Japan's modern-day industrial and corporate productivity. (Ouchi, 1981)

The kind of "just-in-time" collaborative teaming and group problem solving process highlighted in this Japanese cultural example provides some practical insights that might be of use to stakeholders in similar kinds of leading and learning communities (such as schools) who may also find themselves grappling at various times and in specific circumstances with the challenge of needing to find creative ways to deal with pressing, real-world organizational problems. Of course, in contrast to the socially-ingrained collaborative teaming culture that has evolved over centuries in eastern countries such as Japan, societies in the west for the most part are still very much influenced by the allure of rugged individualism and the "competitiveness" that is often viewed as an integral part of organizational life. Thus, in attempting to apply the Japanese model of collaborative teaming and group decision making to various kinds of organizational leading and learning communities in the west, this fundamental difference in cultural perspective must be taken into account. One particularly intriguing aspect of applying this kind of group-centered, organizational team learning and decision making process to organizations in western cultures involves the mental shift many western-style organization members may have to make if they are to become proficient in learning how to work together as a group for the organization's common good. This mental shift essentially involves moving from a predominantly individual emphasis on "competition" (either explicit or implicit) to a new focus based on an understanding of the mutual payoffs that can result from “collaboration”. Indeed, as human cultures have continued his- torically to expand and evolve in complexity over time, this general move away from a predominantly "win-lose” (zero sum) competitive mind-set towards one that affirms the "win-win" (non-zero sum) payoffs realizable through collaboration has been recognized by some cultural evolutionists to be a defining characteristic reflecting the overall trajectory of human cultural “organizing” progress. (Wright, 2000)

As a general method of collaborative teaming practice, this same sort of coming together as a communal group to confront tough problems and to deliberate various options to arrive at agreed-upon, practical solutions is integral to the design of the multimedia case project effort. Of course, in entrenched school situations such as the middle school case reported here, the challenges are more difficult as the stakeholders have reached a point where the problem has evolved into an intractable dilemma-due, in large part, to the entrenched socio-politics impacting the situation. And, to be sure, a central aspect of the middle school community case dilemma highlighted in this article involved the intense "competitiveness" existing between and among various stakeholders and stakeholder groups at this middle school as a result of the multiple perspectivist clashes caused by stakeholders' conflicting values and beliefs regarding instructional change. In an important way, then, stakeholders' immersion in their own multimedia case production team efforts became a creative means to assist stakeholders in making a fundamental "mental shift" in their leadership thinking-from thinking of themselves as mutual adversaries to thinking of themselves as potential collaborators in a new and different kind of problem-solving enterprise. In particular, the members of this middle school case team, as they progressed through the project's various case development and analysis activities, began to mentally distinguish in their own minds between the kind of self-interest driven "opportunistic thinking" that had previously characterized many team members' beliefs and behaviors (and which were so clearly highlighted during the filming of case scenes) and the kind of "communal stewardly thinking" that stakeholders as a team would have to adopt if they were to be able to effectively analyze and make sense of their organizational dilemma. And, when properly internalized, this kind of "communal stewardly thinking" can become a powerful enabling impetus through which stakeholders can begin to "work together in concert" to decide collaboratively what actions they need to take and how they will be implemented. This idea of "working together in concert" itself directly implies that stakeholders functioning cohesively as a leading and learning community must find creative ways to respond to tough problems and to agree on what they are going to do and how they are going to do it. As Gerzon (2006) explains, "The notion of 'working in concert' captures the synergy of many people, each doing what they do best. The importance of 'working in concert' is that it is a holistic approach to action [emphasis added]. It covers everything from the initial convening of the stakeholders all the way through decision making and implementation.” (Gerzon, 2006: pp. 198-199).

Intriguingly, stakeholders' intensive immersion in case production activities over the course of the two-year collaborative project served to trigger a new sense of shared leadership responsibility among participants for their collaborative case development and analysis endeavor, resulting in the distribution of leadership roles and problem-solving responsibilities more evenly across all stakeholders involved. This kind of evolving distributed leadership stance adopted and refined by stakeho- 
lder participants as a group as they worked on case production tasks over the course of the project was noted by university team specialists as an important organizational learning dividend emerging from overall project activities. This notion of stakeholders consciously evolving and fine-tuning their own distributed leadership stance in relation to the context-specific work at hand in leading and learning organizations (such as schools) recognizes and affirms the centrality of the actual leadership practices stakeholders engage in (as opposed to discrete leadership roles, processes, and structures), as well as the ongoing, multiple leadership interactions occurring between and among stakeholders as they enact these practices, as important proximal causes or determinants of measurable instructional improvement (Spillane, 2006: pp. 93-94).

\section{Case Project Results Provided Some Evidence Suggesting Organizational Case Learning Has Promise as an Alternative Staff Development Tool for Building School Community Capacity for Critical Reflection and Transformative Leadership Action}

The overall case development and analysis process, including case team members' collaborative efforts in developing their "video mark" video frame analyses and Reflective Decision Making short- and long-term school community action plans, became for stakeholder participants an important catalyst for learning how to think differently and work together in new ways. Through their involvement in these case development and analysis activities, team members gradually changed the way they mentally looked at their dilemma situation and their perspectivist differences. Within their "video mark" frame analyses, case team members could mentally zoom in on individual "critical incidents" occurring in the case situation as a way to better analyze perspectivist belief clashes between and/or among various stakeholders and stakeholder groups. This, in turn, enabled team members to begin to discern (at a deepstructural analytic level) the underlying organizational root causes of their dilemma challenges that were fueling these surface-level perspectivist clashes and, as a result, team members could begin to develop more insightful understandings about the origins of their dilemma. In effect, this process enabled team members in a more informed way to begin to address the central organizational sense making question: why or how did we get to where we currently are now as an organization? The critical reflective conversations team members engaged in were like a multi-perspectivist "360 degree walk around" their dilemma situation, enabling team members to critically view and examine their organizational dilemma challenges from multiple angles at once (reminiscent, in an analogous way, of a similar kind of "simultaneous, multiple perspectives" visual mode of representation which can be found in some graphic art- notably, in some of Pablo Picasso's paintings). Case team members were then able to leverage these "analytic insights"-which they were able to collaboratively evolve and crystallize during case scenes filming and during their "video mark" frame analyses - to begin the important process of forging a coherent, communal vision of "who we are and where we want to be going as a middle school leading and learning community".

One important benefit emerging from stakeholders' sustained efforts during multimedia case development activities was that case team participants began to look with new eyes on their collective perspectivist differences as a potent, valuable asset rather than as a liability. These differences came to be viewed no longer by these middle school stakeholders as insurmountable roadblocks, but as opportunities that enabled stakeholders to engage in new kinds of critical reflective conversations. And, these conversations were found to be useful for engendering new collegial insights on the possibilities of communal leadership, and for helping school stakeholders rediscover themselves as a coherent and purposeful organizational leading and learning community. A key breakthrough insight team members realized through their involvement in the multimedia case development process was that robust stakeholder perspectivist differences - and the group's collective ability to recognize, respect, and leverage those differences to inform and energize their critical reflective conversations-was, in itself, a nuanced and valuable organizational core competency that these middle school stakeholders as a group could develop, refine, and optimize in their own way to inform their collaborative decision making.

The critical reflective conversations team members engaged in during the case development and analysis process also represented for these middle school stakeholders a new way of interacting and communicating-one in which stakeholders became actively involved as a group in examining, analyzing, and using their own situational data to collaboratively make sense of their dilemma challenges and to brainstorm realistic action plan strategies. The critical reflective and multi-perspectivist insights stakeholders were able to generate during their team conversations concerning the root causes of their organizational dilemma challenges provided a new degree of analytic clarity which empowered stakeholders to be able to work together in new ways as a communal leadership team to fashion specific sets of short- and long-term action strategies to move their school community forward. In this sense, team members' critical analytic insights on the root causes of their dilemma challenges became the catalyst for envisioning and realizing transformative leadership action-leadership action strategies that when implemented could result in meaningful organizational improvement. This new way of interacting and communicating with each other through focused conversations grounded in examining and analyzing the team's own organizational data also played an important role in building an evolving sense of collegial trust among stakeholder team members, both in the process itself and in their own analytic abilities. The literature on organizational learning and development has affirmed the value of this practice of encouraging as many organization members as possible to become actively involved in scrutinizing an organization's data and holding conversations about their observations as a means for generating a wide array of organizational insights and potential creative solutions to problems. As Wheatley (1992) has noted, "a multiplicity of interactions [among diverse organization members] can give a genuine richness to the data that is lost when we restrict information access to only a few people . . . an organization swimming in many interpretations can then discuss, combine, and build on them; the outcome of such a process has to be a much more diverse and richer sense of what is going on and what needs to be done" (Wheatley, 1992: p. 65).

Moreover, middle school case team members began to see parallels on how the same critical reflective analysis skills they were acquiring during these project-related case development experiences (i.e., new collaborative techniques for intensively 
mining and leveraging stakeholders' own situational data to achieve breakthrough insights to inform their organizational improvement action strategies) could also be applied directly within team members' own ongoing instructional teaming practices. These middle school educators and community members, in effect, were learning about the payoffs of critical reflective analysis through immersion directly in the teaming process. In this regard, the overall multimedia case development process served as a kind of organizational learning incubator for assisting team members in developing a genuine team analytic mind-set. A central observation noted and confirmed repeatedly by university project specialists (as well as by school-based team members themselves) as they worked on case development activities with stakeholders at this school and others in the region was the emerging organizational learning insight that school stakeholders had to actually come together in an intensive collaborative effort and experience first-hand the payoffs achievable through engaging in sustained critical reflective analysis of their own situational data (including spending time reflecting on what and how they were learning) to be able to fully appreciate and embrace teaming as a viable tool that could enhance their ongoing professional practice. Stakeholders' overall project experiences as a "case production team" served to provide them, in effect, with an immersive experiential template for how they could begin to work together constructively in their school community instructional teams. Thus, an important outgrowth of the multimedia case development process was that stakeholder participants (teachers, administrators, and parents in this middle school community) began to feel comfortable with and believe in themselves and their analytic abilities as a team. As a result, case development team members as a group began to accept the idea of team-centered, data-driven critical reflective analysis as a viable form of professional and organizational practice- - collaborative practice that could reap tangible dividends and lead to real payoffs for the whole school organization.

Furthermore, immersion in the multimedia case development process was for these middle school stakeholder participants an alternative kind of staff development experience. Participation in the multimedia case development and analysis project was seen as an immersive and more dynamic form of organizational learning that could replace some of the more traditional kinds of staff development that teachers were familiar with. Most importantly, multimedia case project activities provided a unique means for stakeholders to leverage their own context-specific dilemma challenges in a constructivist-experiential manner to discover new reservoirs of collaborative team energy and engage in organizational learning in a new way. Through participating in the overall process of developing, refining, and producing their multimedia school leadership case, these middle school educators and community members were able to "tap into" their own collaborative potential for critical reflection and transformative leadership action. And, in so doing, these stakeholders were able to prove to themselves that working together as a purposive, collaborative team to solve organizational challenges has advantages over simply relying on the sum of individual effort-and that multi-stakeholder teaming (when properly developed and grounded in a genuine collaborative team mind-set) can indeed lead to more effective and sustainable organizational improvement results.

Finally, as a caveat, it is important to note that the organizational learning and development payoffs these middle school case team stakeholders realized as a result of their project experiences were substantive and school community-enhancing, but were also hard won and came with a price. The multimedia case development process described in this project report is certainly one that is time-consuming and effort-intensive-one that requires a high level of sustained commitment by all participants involved (teachers, department chairpersons, campus-level administrators, parents, school district central office personnel, and partnering university specialists). Moreover, this kind of multimedia case development endeavor can only be genuinely successful if backed by strong and continuous administrative and motivational support from the school principal and key district central office personnel. Even with these supportive elements in place, however, for a school community to be able to engage effectively in this kind of alternative staff development endeavor a fundamental key ingredient must be in place and subscribed to by all school community participants. That key ingredient is a conscious and proactive willingness on the part of all stakeholders involved to experiment, to take risks, and to explore and extend the limits of their own organizational learning through thinking differently and working together in new ways. This kind of adventuresome spirit was captured well by one of the school's veteran eighth-grade teachers who, reflecting near the end of the two-year case project on her collective experiences as a member of the case production team, noted the sense of professional renewal-and the sense of new responsibility—she felt toward her grade-level colleagues and her school community, as well as the sense of renewed commitment she felt toward her own teaching: "As everyone on our case production team knows so well by now, I was the real skeptic in the group. And, even now, I'm not sure that teaming can solve all of our school challenges. But I can say that this project has caused me to expand my horizons. I now understand better the value of taking the time to listen to and to really try to understand others' views. It's not just about agreeing or not agreeing with others. As we've learned in our case production work, teaming goes well beyond that. It's really about the bonds of respect that develop between and among people who have decided to come together to identify and achieve a focused goal. You don't have to agree on everything to succeed as a team, but you do have to agree on and want to achieve a common goal. When we first began this project, we weren't even sure what a multimedia case was. But the idea grew on us, and we made it our own. As we became more and more involved in the production effort (and it really was an effort), this case project became our project. What seemed at first like a lot of work became more enjoyable as we went on. For me, the teaming itself has become something that I now value-the teaming itself has become a new way.” Similar sentiments regarding both the nature of the work and the individual and team learning value of case project experiences were voiced by other stakeholder members of this middle school case production team as they participated in case project concluding activities.

Perhaps it was the principal who best summarized the collective feelings of her middle school case team colleagues when, at a spring school stakeholder gathering to celebrate the project's completion, she remarked, "I feel much better about our prospects for tackling our school improvement issues now than I did two years ago. Our student learning challenges in science and math are still very real, and we will have to continue to work very hard to achieve measurable progress, and to sustain those incremental improvement results. But our case team ex- 
periences have helped us learn how to become a team, how to work together as a group to develop shared vision and purpose. We now know that if we put our minds to it we can set clear goals and work together as a team to achieve those goals. We now know how it feels to be a functioning team-we've experienced the payoffs of collaborative teaming. What we need to do now is to continue to work together in our grade-level and campus improvement teams to internalize this teaming mind-set further and make it a permanent part of our school's instructional teaming culture." As university production team members witnessed again and again in partnering with different groups of campus-based stakeholders during multiple case development project efforts at different schools over several years, the organizational learning payoffs of coming together as a multi-stakeholder team to experiment, to take risks, and to explore and extend the limits of your own and your team's organizational learning potential can yield valuable rewards - not the least of which can be more informed and insightful understandings of the new opportunities and renewed levels of commitment that can be realized through the teaming process itself. In short, as school stakeholders who are confronting intractable, real-world dilemma challenges elect to become multimedia case developers and embark on this kind of alternative shared learning experience, these same school stakeholders-working together as a group to effect meaningful change and improvement in their school community-must be willing to suspend their prejudices and disbelief and place their faith, even if conditionally, in the promise, prospects, and payoff potential of collaborative teaming.

\section{Conclusion}

The multimedia case collaborative project efforts engaged in by middle school stakeholders and university specialists and reported in this article represent one case installment in a larger body of organizational cases developed over more than a decade of intensive research and development work focused on providing meaningful organizational learning and development opportunities to K-12 educators. A unique feature of this school-university collaborative project involves leveraging the power of multimedia technology to engage school stakeholders directly in addressing their own context-specific school leadership dilemma challenges through sustained immersion in a constructivist multimedia case production and analysis team learning experience. The multimedia case development design utilized in this project reflects a creative integration of three central design elements-dramatic theatre, cinematography, and computer-based simulations - used in combination to create a unique organizational team learning experience for school stakeholder participants.

The multimedia case learning work described above falls within and is supported by a rich tradition of creative research and development in the use of computer-based gaming and simulations in education. Indeed, the widespread use today of educational games and simulations in $\mathrm{K}-12$ and university classrooms to enhance teaching and learning in both synchronous and asynchronous (i.e., face-to-face and interactive webbased) teaching environments in a variety of applied content areas (such as in math, science, economics, and engineering, to name a few) itself draws on the commercial success of a number of popular online games and simulations that have evolved in the past decade, some of which have found their way into educational classrooms (e.g., SimCity, Civilization, and Oregon Trail). As a direct result of the evolving commercial development and popular use of gaming and simulations, online virtual worlds such as The Sims and Second Life are now being used with increasing regularity by educators as teaching and learning tools to engage students in sophisticated simulated environments in ways that dynamically frame concepts and immerse learners in interactive worlds of constructivist exploration and learning. Moreover, a growing body of research on the educational use of computer-based games, simulations, and related multimedia technologies has emerged in the past several years exploring multiple utilization issues as well as factors influencing learning effectiveness (Mayer, 2005). This research has examined the impact of using multimedia simulations within a broad array of instructional approaches and interactive learning contexts. The organizational case learning R\&D project work profiled in this report seeks to contribute to the literature on the use of computer-based simulations to enhance learning in the social sciences (Petrovic \& Brand, 2009; Shiratori, Arai, \& Kato, 2005) through focusing specifically on the application of multimedia simulation design to real-world challenges associated with education stakeholders' organizational leading and learning development in K-12 school communities.

Importantly, the case development and analysis activities engaged in by middle school stakeholders participating in the organizational case development project reported here reflect a conscious effort to move away from the kinds of staff development programs typically available to K-12 campus teachers and administrators (e.g., "ad hoc" programs that tend to focus, often in a somewhat piecemeal way, on discrete, stand-alone aspects of classroom teaching and student learning performance). In contrast to these kinds of traditional staff development offerings, this project utilizes a more targeted and holistic approach that emphasizes the value of leveraging the power of multimedia simulation design to immerse school leaders directly, in an in-depth way, in their own school organizational data through involving these stakeholders in the constructivist re-creation and analysis of their own context-specific leadership dilemma situations via organizational case learning. At the heart of this multimedia case learning approach is the intent to create an immersive and data-rich interactive team learning environment within which school community stakeholders (teachers, administrators, parents, central office personnel, and other interested stakeholders) can engage together in the constructivist re-creation and critical analysis of their own context-specific dilemma situations as a means to reframe these dilemma situations and generate transformative, action-based solutions to effectively move their school communities forward.

Collective efforts of middle school educators and community members participating in this case development project enabled stakeholders to more critically examine their perspectivist differences and to carefully analyze the root causes of their dilemma situation. As a result of their multimedia organizational case learning experiences, these stakeholders were able to forge a new team mind-set for generating new leadership insights to positively inform their real-world instructional decision-making. Through leveraging the power of multimedia technology and an adventurous "case production team" spirit (as well as an acquired "Avatar-like" cultural respect-a la Grace Augustine - for the organizational case learning benefits of comprehending differences and building multi-stakeholder understandings), these middle school stakeholders were able to dis- 
cover new organizational purpose and direction and develop a more insightful appreciation for the school improvement payoffs of collaborative teaming.

\section{REFERENCES}

Cameron, J. (2009). Avatar [Motion picture]. Los Angeles, CA: Twentieth Century Fox Film Corporation and Dune Entertainment, LLC.

Civilization (2012). Online multiplayer game/simulation (originally created in 1991). URL (last checked 10 October 2012).

http://www.freegameempire.com/games/Civilization

Gerzon, M. (2006). Leading through conflict: How successful leaders transform differences into opportunities. Boston, MA: Harvard Business School Press.

Jacobs, R. W. (1997). Real-time strategic change: How to involve an entire organization in fast and far-reaching change. San Francisco, CA: Berrett-Koehler Publishers.

Love, N., Stiles, K. E., Mundry, S., \& DiRanna, K. (2008). The data coach's guide to improving learning for all students: Unleashing the power of collaborative inquiry. Thousand Oaks, CA: Corwin Press.

Mayer, R. E. (2005). The Cambridge handbook of multimedia learning. Cambridge: Cambridge University Press. doi:10.1017/CBO9780511816819

Oregon Trail (2012). Online game/simulation (originally created in 1974). URL (last checked 10 October 2012).

http://www.freegameempire.com/games/Oregon-Trail

Ouchi, W. G. (1981). Theory Z: How American businesses can meet the Japanese challenge. New York: Addison-Wesley Publishers.

Petrovic, O., \& Brand, A. (2009). Serious games on the move. Berlin:
Springer-Verlag/Wien. doi:10.1007/978-3-211-09418-1

Second Life (2012). Online virtual world simulation (originally launched: 23 June 2003). URL (last checked 10 October 2012). http://secondlife.com/

Seymour, J. (1995). Japan, Vol. 1: The electronic tribe [VHS]. Four-part PBS documentary series. Chicago, IL: WTTW/Coronet Film \& Video.

Shiratori, R., Arai, K., \& Kato, F. (2005). Gaming, simulations, and society: Research, scope, and perspective. Tokyo: Springer-Verlag. doi:10.1007/b138103

SimCity (2012). City-building online simulation/video game (first released: 3 October 1989). URL (last checked 10 October 2012). http://www.simcity.com/en_US

Smith, J. A., Flowers, P., \& Larkin, M. (2009). Interpretative phenomenological analysis. London: Sage Publications.

Spillane, J. P. (2006). Distributed leadership. San Francisco, CA: Jossey-Bass Publishers.

The Sims (2012). Online life-simulation game series. URL (last checked 10 October 2012) http://thesims.com/en_US/home

Van Manen, M. (1990). Researching lived experience (2nd ed.). Albany, NY: State University of New York Press.

Weisbord, M. R. (1992). Discovering common ground: How future search conferences bring people together to achieve breakthrough innovation, empowerment, shared vision, and collaborative action. San Francisco, CA: Berrett-Koehler Publishers.

Wheatley, M. J. (1992). Leadership and the new science: Learning about organization from an orderly universe. San Francisco, CA: Berrett-Koehler Publishers.

Wright, R. (2000). Nonzero: The logic of human destiny. New York: Pantheon Books. 